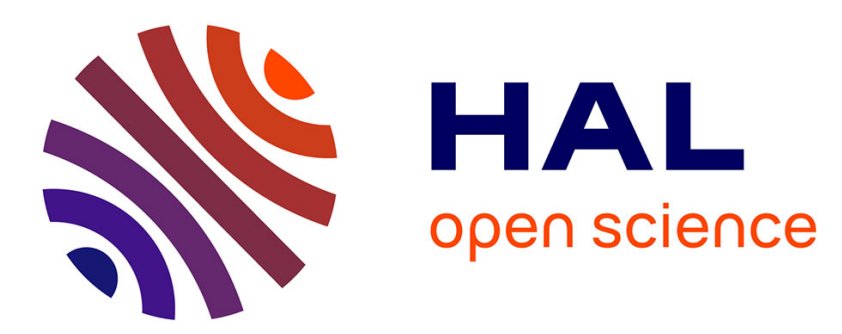

\title{
L'émergence du concept de discours indirect libre et la représentation des pensées: quand le terme opacifie la réalité
}

Olga Artyushkina

\section{- To cite this version:}

Olga Artyushkina. L'émergence du concept de discours indirect libre et la représentation des pensées: quand le terme opacifie la réalité. Revue des études slaves, 2012, Mélanges offerts à Jean Breuillard. hal-01264517

\section{HAL Id: hal-01264517 \\ https://hal.science/hal-01264517}

Submitted on 3 Feb 2017

HAL is a multi-disciplinary open access archive for the deposit and dissemination of scientific research documents, whether they are published or not. The documents may come from teaching and research institutions in France or abroad, or from public or private research centers.
L'archive ouverte pluridisciplinaire HAL, est destinée au dépôt et à la diffusion de documents scientifiques de niveau recherche, publiés ou non, émanant des établissements d'enseignement et de recherche français ou étrangers, des laboratoires publics ou privés. 


\section{L'émergence du concept de discours indirect libre et la représentation des pensées Quand le terme opacifie la réalité}

$\mathrm{D}$ ANS CET ARTICLE, nous allons nous intéresser à l'émergence du concept de discours (ou style) indirect libre, connu en russe sous sa dénomination la plus courante nesobstvenno-prjamaja rečl ${ }^{1}$ (« discours improprement direct »). Le phénomène du discours indirect libre (désormais : DIL) est relativement récent : on cite toujours sa modernité comme un de ses traits caractéristiques. En effet, son emploi dans l'écriture est devenu massif grâce à la naissance du roman réaliste et psychologique, qui a connu un grand essor au XIX siècle en Europe.

Rappelons que le DIL se définit comme une forme originale du discours rapporté qui conjugue les caractéristiques des discours direct et indirect : tout comme le discours direct, il préserve les particularités des paroles rapportées, mais les formes personnelles (verbes, pronoms) sont transposées comme au discours indirect. Au cours de notre recherche, nous avons établi une grille d'analyse d'inspiration pragmatico-énonciative qui nous sert à confirmer (ou infirmer) l'appartenance des énoncés au DIL : premièrement, la situation pragmatique doit être compatible avec l'émission d'un discours ; deuxièmement, ce discours doit être formulé dans les termes que l'on interprète comme relevant de l'instance énonciative rapportée ${ }^{2}$.

Malgré une riche littérature consacrée au DIL en tant que catégorie linguistique et procédé stylistique ${ }^{3}$, sa définition formelle est souvent insatisfaisante. Lorsqu'on s'intéresse à ce phénomène linguistique complexe, on ne peut que s'étonner du manque de cohérence dans l'emploi du terme même. En effet, cette étiquette recouvre des formes langagières extrêmement diverses, ce qui, de fait, brouille toute compréhension du phénomène et opacifie les réalités langagières qu'il faudrait analyser plus attentivement, afin de ren-

1. D'autres termes existent également : svobodnaja kosvennaja reč' (GVOzDEV 1952), svobodnyj kosvennyj diskurs (PADUČEVA 1996), nesobstvenno-avtorskaja reč' (SOKOLOVA 1968).

2. Cette grille préalable à toute analyse du présumé DIL va dans le sens des recherches de M. DE MATTIA-VIVIÈs $2006: 37-43$.

3. Cf. les travaux de PAduČEva, ŠMID, Rigato dans la bibliographie. 
dre compte des traits distinctifs entre le DIL et les autres formes d'énonciation qui ne s'inscrivent pas stricto sensu dans la dichotomie célèbre d'Émile Benveniste entre « histoire » et « discours ».

Cet article se donne les objectifs suivants : premièrement, rendre compte de l'emploi du terme «DIL » et, deuxièmement, mettre en évidence la spécificité du DIL représentant les pensées et, plus largement, la difficulté d'une telle représentation dans le récit de fiction.

\section{Un aperçu des définitions du discours indirect libre : critique et problématique}

Dans les lignes qui suivent, nous montrerons d'abord quels sont les différents phénomènes langagiers désignés par DIL et, ensuite, nous expliquerons les raisons d'une grande hétérogénéité dans le corpus qui est donné à titre d'illustration.

\subsection{Le discours indirect libre : quelle(s) réalité(s) langagière(s) ?}

Nous commencerons par faire une mise au point préliminaire qui concerne la définition du terme «DIL », car c'est là que surgit la première difficulté. La comparaison des exemples de DIL fait constater que les structures du discours rapporté entendues par ce terme divergent. Observons deux exemples :

[1] Вот теперь трактирщик сказал, что не дам вам есть, пока не заплатите за прежнее.

(Н. Гоголь, Ревизор)

[Mot à mot] : l'aubergiste vient de dire que je ne vous donnerai pas à manger, tant que vous n'aurez pas payé les repas précédents.

[2] Мазепа, в горести притворной, к царю возносит глас покорный. «И знает Бог, $u$ видит свет: он, бедный гетман, двадиать лет изарю служил душою верной; его щзедротою безмерной осыпан, дивно вознесен [...]» (А. Пушкин, Полтава, exemple de la grammaire académique de 1954, pp. 428-429)

Le premier exemple est souvent cité ${ }^{4}$ : la structure syntaxique, attestée déjà en vieux russe, introduit le discours d'autrui (ici et plus loin, les italiques sont de nous) d'une manière « libre». Le terme russe « nesobstvenno-prjamaja reč' $\gg$ est en soi assez bien choisi pour qualifier ce type de structure, car il fait figurer le terme de discours direct dont la primauté est soulignée par Vološinov. Pour Uspenskij ${ }^{5}$, cet exemple est un cas représentatif du DIL et ne doit pas être confondu avec le discours intérieur représenté.

Cependant, le sens le plus communément admis par le DIL s'applique à ses manifestations libérées de toute subordination, avec les formes personnelles repérées par rapport au récit, comme dans l'exemple [2], qui illustre le DIL classique, ou traditionnel, représentant les paroles rapportées ; on réservera le nom de « discours semi-direct» à la structure du premier exemple. Il

4. Exemple cité dans PEŠKOvsKiJ 1920/1956 : 465-466, repris dans VološInOv 1929/1998 : 454 ; GVOZDEV 1965 nomme ce type de structure «poluprjamaja $r e c^{\prime} »$ » (le discours semi-direct).

5. USPENSKIJ $2000: 62$. 
est évident que l'on ne pourra réunir [1] et [2] dans la même catégorie linguistique ; il s'agit de structures au fonctionnement énonciatif différent : dans [1], le repérage énonciatif des formes personnelles est effectué sur l'énonciateur rapporté ; dans [2], ces marques personnelles sont alignées sur le récit.

Cet amalgame entre le discours semi-direct et le DIL traditionnel a été fait en partie pour des raisons chronologiques : c'est le discours semi-direct qui est apparu historiquement en premier comme troisième forme originale du système du discours rapporté, alors que, par exemple, en ancien français la forme alternative avait déjà les traits du DIL dans son emploi en français contemporain ${ }^{6}$. La variante du DIL équivalente en russe n'apparait qu'avec les fables de Krylov (1769-1844) ${ }^{7}$, puis devient procédé stylistique populaire grâce à Puškin.

\subsection{Le discours indirect libre comme procédé d'introspection psychologique}

Le roman dit « réaliste », " psychologique », introduit une nouvelle pratique dans l'écriture et aussi de nouvelles motivations : on s'intéresse davantage à la vie intérieure du personnage, aux motivations de son comportement. Naturellement, cette psychologisation de l'écriture crée l'intérêt pour la représentation des émotions, sentiments, pensées d'autrui et sa perception en général. Ainsi, le DIL classique devient un procédé apprécié pour rapporter le discours d'autrui en même temps que l'on cherche à représenter le monde des pensées, qui ne sont pas de nature entièrement verbale et dont la représentation au DIL est soumise aux contraintes que les paroles rapportées ignorent. Mais hormis quelques exceptions (voir Uspenskij), les travaux sur le DIL en russe incluent, par amalgame, dans la catégorie «DIL » toutes sortes de procédés de l'introspection psychologique du récit de fiction.

Ce manque d'attention pour le DIL représentant les pensées peut s'argumenter par le fait que le discours non extériorisé peut être formulé intérieurement d'une manière identique à celui qui est adressé à un véritable interlocuteur-destinataire ; dans ce cas-là, on peut ajouter une incise « se dit-il », «pensa-t-il ». Mais ce test ne réussit pas toujours, surtout lorsqu'il s'agit des états mentaux complexes, comme c'est le cas dans l'exemple suivant :

[3] Он стоял как вкопанный... Мучительная мысль сверлила его мозг : эта ложа, на которую он дал слово не смотреть... Княгиня сидела в ней, ее розовая ручка покоилась на малиновом бархате, ее глаза, может быть, часто покоились на нем, - а он даже и не подумал обернуться, магнетическая сила взгляда любимой женшины не подействовала на его бычачьи нервы - о, бешенство! - он себе этого никогда не простит ! Раздосадованный, он пошёл по тротуару ${ }^{8}$.

(М. Лермонтов, Княгиня Лиговская, exemple de la grammaire académique, 1954, p. 430)

6. Cf. sur l'histoire des structures syntaxiques du discours rapporté VoLOŠINOV 1929/1998 : 468-478.

7. Cf. J. VeYrenc 1982.

8. L'exemple donné par la grammaire s'arrête aux paroles «knjaginja sidela v nej», qui rendent les propos assez ambigus en l'absence du contexte de droite plus large que nous donnons ici. 
Il nous semble important de souligner que lorsqu'il s'agit de la représentation des pensées, le rapport de la forme linguistique à l'objet représenté n'est pas identique à celui de la représentation des paroles. On pourrait résumer la différence essentielle entre DIL des paroles en [2] et DIL des pensées en [3] de manière suivante : si, dans [2], nous avons effectivement une représentation du discours d'autrui, facilement restituable dans sa forme d'origine, dans le [3], il s'agit déjà d'un grand réaménagement de la prise de conscience du personnage - seule une partie du présumé DIL peut être vraisemblablement verbalisée. Notons que si l'on coupait cet exemple aux paroles « knjaginja sidela $v$ nej », comme le fait la grammaire (nous avons donné un contexte plus large), on ne pourrait alors nullement interpréter ce passage comme relevant du DIL. Coupé de sa partie chargée en éléments expressifs et discursifs à proprement parler (" a on daže... ne prostit »), l'extrait s'interpréterait alors comme le récit à point de vue (ou « psycho-récit », terme proposé en narratologie par D. Cohn), les verbes au prétérit assurant la continuité du plan narratif (le DIL, lui, est associé au temps présent). On voit ainsi la nécessité absolue d'avoir un contexte suffisamment large pour une interprétation correcte du discours intérieur représenté.

D'autres définitions du DIL ne font pas du discours intérieur un cas à part : les définitions des dictionnaires des termes linguistiques ${ }^{9}$ de $\mathrm{O}$. Axmanova et de D. Rozental' tracent un signe d'égalité entre le monologue intérieur, la description des émotions, les impressions, etc., et le DIL. Les exemples donnés ne sont pas toujours très convaincants :

[4] Николай Ростов отвернулся и, как будто отыскивая чего-то, стал смотреть на даль, на воду Дуная, на небо, на солнце. Как хорошо показалось небо, как голубо, спокойно и глубоко! Как ласково-глянцевито блестела вода в далеком Дунае!

(Л. Толстой, Война и мир, exemple de D. Rozental', 1976 : 206)

Il ne s'agit ici nullement du discours : en effet, il n'est pas envisageable que le personnage, Nikolaj Rostov, se décrive intérieurement dans les termes présents la beauté du spectacle offert par la nature ; il s'agit ici simplement de la perception d'autrui sans qu'il y ait de discours possible. La représentation de la conscience est ici très caractéristique du style tolstoïen : le narrateur manifeste sa présence par le caractère très construit des perceptions. Une autre marque supplémentaire qui éloigne ce passage du plan du discours est le temps des verbes : pokazat'sja " paraître » et blestet" « briller», employés au prétérit, ne créent pas de rupture avec le plan du récit pour basculer dans le discours. Ainsi, la situation pragmatique du discours est peu concevable et les termes choisis pour représenter la perception sont manifestement ceux du narrateur, ce qui nous conduit à exclure cet exemple de la catégorie de DIL.

On peut dire que le DIL des pensées est sa variante la plus investie par le narrateur, car « la parole origine [le discours qui est représenté - O.A.] a un statut hypothétique ${ }^{10} \gg$ et l'énoncé récupéré, au statut purement fictif, néces-

9. O. Axmanova $1966: 239$, D. RozentaL' $1976: 205-206$.

10. De Mattia-Viviès $2006: 39$. 
site une articulation supplémentaire pour être accessible au lecteur. En ce qui concerne notre jugement sur la vraisemblance de la forme des pensées d'autrui, elle dépend entièrement de l'idée qu'on se fait du discours intérieur ; cette idée varie en fonction de l'époque : si l'exemple [3] pouvait paraître tout à fait naturel pour le lecteur contemporain de Lermontov, aujourd'hui, notre idée sur la pensée est associée au discours endophasique ; par conséquent, le [3] parait trop artificiel pour une représentation vraisemblable du discours intérieur. Ainsi, il convient de s'interroger sur la nature du processus de la pensée et les différentes étapes qui la constituent.

\section{La représentation des pensées : que dit-on à soi-même et comment ?}

Nous avons vu que la mise en signes linguistiques de la pensée tient obligatoirement compte de l'exigence de clarté de la part du lecteur, ce qui constitue une particularité du schéma communicatif du DIL des pensées : la forme du message doit considérer les besoins du lecteur, qui se trouve en position de second destinataire du discours intérieur ; c'est pour le lecteur qu'on précise plusieurs détails, car l'énonciateur lui-même ne les mentionnerait pas intérieurement. Pour comprendre le fonctionnement du DIL des pensées qui, finalement, construit plus qu'il ne rapporte, une réflexion sur le processus d'articulation du discours, sur la part du verbal et du non verbal s'impose. À ce sujet, les remarques d'Ann Banfield dans son travail «Phrases sans parole ${ }^{11}$ » sont intéressantes : on peut distinguer dans la conscience deux niveaux - réflexif et non réflexif, ce qui correspond dans la représentation de la pensée à la différenciation entre le caractère verbal et non verbal de l'état mental.

Rappelons brièvement à quoi correspondent ces deux niveaux de conscience. Un savoir non réflexif est un savoir qui se trouve au niveau inconscient et qui correspond au niveau non verbal mais il peut devenir à son tour réflexif, accessible à la conscience si l'on nous interroge dessus ; Russel (1940 : 58-59) en donne l'exemple suivant :

\footnotetext{
Imaginons que, vous promenant un jour de pluie, vous apercevez une flaque devant vous et que vous l'évitez. Il y a peu de chances que vous vous soyez dit : "Voici une flaque d'eau; mieux vaut ne pas marcher dedans. » Mais si quelqu'un vient vous demander : « Pourquoi avez-vous fait ce détour? » vous répondrez certainement « parce que j'ai voulu éviter de marcher dans la flaque ». C'est donc que, rétrospectivement, vous saviez : vous avez eu une perception visuelle $[\ldots]$ et $[\ldots]$ vous exprimez ce savoir en mots.
}

Revenons à notre exemple [3] pour comprendre la représentation de l'état mental dans lequel se trouve le personnage : il s'agit de la remémoration, d'une prise de conscience d'un savoir ; l'extrait «èta loža ... sidela v nej» constitue un savoir existant au niveau de la perception visuelle, non verbale 
(le sujet de conscience ne se commente pas tout ce qu'il voit), mais cette information se trouve confrontée à un contexte nouveau, qui amène le sujet à reconsidérer son savoir : non réflexif, il devient réflexif. Le personnage voit une femme, la dame qui était assise dans la loge voisine et dont il ne pouvait voir le visage, et au moment où celle-ci quitte le théâtre, il reconnaît en elle la princesse Ligovski, dont il est amoureux. C'est donc en revoyant les images d'une « inconnue », qu'il s'était interdit de regarder par défi, trouvant que son intérêt tournait à l'obsession, qu'il se met en colère contre son insensibilité, n'ayant pas su reconnaître, sentir la présence d'un être cher.

Ainsi, le savoir cumulé au niveau non réflexif, essentiellement sous forme visuelle, se trouve verbalisé, cette verbalisation étant motivée par un contexte nouveau (ici : émotion de colère contre soi-même). Cependant, comme nous l'avons déjà noté, cet exemple ne correspond pas d'une manière satisfaisante à notre critère de réalisme langagier du lecteur contemporain : les détails des scènes ressorties des «archives » de la mémoire visuelle («eё malen'kaja ručka pokoilas' na malinovom barxate [...]») sont trop précis et agencés d'une manière trop construite pour un discours qui présuppose une certaine spontanéité. Toutefois, dans son ensemble, l'exemple [3] représente une configuration qui sert de matrice pour la représentation de la pensée : les parties visuelle (point de vue) et verbale (discours) sont soudées pour donner une idée d'un processus de l'articulation de la pensée qui tend vers le discours.

Atteindre le « réalisme » dans la représentation de la parole intérieure est une tâche dont on doit mesurer la difficulté : l'accès à l'inconscient du personnage, effet recherché dès l'apparition de la technique narrative du monologue intérieur, ne peut s'effectuer qu'au prix d'un partiel renoncement à la réalité cognitive du processus. Comme le remarque A. Rabatel (2001 : 80), la représentation de la parole intérieure est sujette à tension « entre une nonclarté psychologique de nature autoscopique et une lisibilité minimale » : en d'autres termes, l'écrivain est obligé de présenter la ramification de la pensée au nom de l'intérêt de son projet artistique et esthétique. L'état mental qui précède tout discours articulé n'a pas de recours aux paroles : la représentation qui en est faite dans le récit est alors due à l'instance énonciative du narrateur. A ce sujet, le concept de «phrases sans parole» (traduction de «unspeakable sentences») proposé par Ann Banfield s'avère appréciable, car il a le mérite de rendre compte des phrases qui, en construisant la représentation de la subjectivité d'autrui, ne créent pas de situation énonciative.

Voyons à présent quelques exemples de représentation de la pensée et de la perception d'autrui dans le récit, afin d'essayer d'entrevoir les frontières du concept de DIL, tâche qui soulève des problèmes d'ordre épistémologique. 


\section{Le continuum des formes représentant les pensées}

Dans notre aperçu de l'histoire du terme de DIL, nous avons vu que le manque de précision dans la terminologie était dû au contexte de son émergence : il était envisagé comme une troisième catégorie intercalée entre le plan du récit et celui du discours. É. Benveniste, conscient des limites de la dichotomie « histoire (récit)-discours », suggérait le discours rapporté comme troisième catégorie. En ce sens, les narratologues ont pris de l'avance sur les linguistes dans leur analyse de la représentation de l'espace perceptif d'autrui en général et de l'univers des pensées en particulier : les travaux de Todorov, Genette, Cohn mettent en évidence les différentes stratégies narratives où le narrateur peut être présent à des degrés divers ; en russe, on peut se référer, par exemple, aux travaux de Baxtin, Vološinov, Uspenskij. L'approche offerte par la linguistique énonciative s'intéresse à la diversité de formes linguistiques qui se trouvent en dehors de la dichotomie « récit-discours ${ }^{12}$ » : par exemple, les acquis de recherches en narratologie ont été mis à profit par A. Rabatel (2001 : 88-89), qui offre une optique nouvelle et propose un terme " point de vue », narratologique à l'origine :

Dans le PDV [point de vue - O.A.], la parole intérieure existe en tant que trace expressive [...] d'un mouvement perceptif et interprétatif qui ne s'extériorise pas par les marques traditionnelles de la parole et de la pensée. [...] le point de vue s'apparente à une sorte de pensée non réflexive, ou pré-réflexive, ou encore, selon la paraphrase de Banfield, à une « conscience spontanée ».

Nous allons voir quelques cas de figure où le point de vue alterne avec le récit, servant éventuellement de préfiguration à la pensée articulée.

\subsection{La conscience non réflexive}

Il s'agit d'un exemple de la représentation de la perception que l'on intègre parfois, à tort, dans la catégorie du DIL ${ }^{14}$, alors que les éléments, ou « traces expressives » appartenant à autrui ne sont pas suffisantes pour poser une situation du discours :

[5] Будочник глядел, кажется, с любопытством, желая узнать, какого чёрта бежит к нему издали и кричит человек. Н. Гоголь, Шинель

[6] Ce pauvre diable d'ouvrier, perdu sur les routes, l'intéressait.

(É. Zola, Germinal, exemple emprunté à A. Banfield 1995 : 301)

\subsection{Les fragments du point de vue intriqués avec le récit}

Un agencement argumentatif particulier entre le récit et les séquences qui comportent des éléments susceptibles de construire un espace énonciatif

12. Sur les formes en dehors de la dichotomie benvenistienne, cf. I. KoR CHAHINE pour le russe, A. Rabatel pour le français, M. De Mattia-Viviès, H. ChuQuet, S. Hanote pour l'anglais ; cf. également Les Actes de la journée d'études «Énonciation et narration 》 du 25 juin 2010, à paraître dans Slovo.

13. Cf. BANFIELD $1995: 293-313$.

14. Pour le commentaire des "faux-DIL", cf. notre thèse de doctorat, pp. 129-133, 137. 
d'autrui peut donner au lecteur l'impression d'assister au dialogue entre le narrateur et la conscience du personnage :

[7] А разве я говорил что дурное про властей? - спросил Коваленко, глядя на него со злобой. - Пожалуйста, оставьте меня в покое. [...] Я не люблю фискалов. Беликов нервно засуетился и стал одеваться быстро, с выражением ужаса на лице. Ведь это первый раз в жизни он слылшал такие грубости.

— Можете говорить, что вам угодно, — сказал он [...]

(А. Чехов, Человек в футляре ${ }^{15}$ )

Les actions « se dépêcher » et «s'habiller» sont d'abord posées par le narrateur et justifiées ensuite du point de vue du sujet de cette action. Deux traces expressives indiquent ici que la phrase qui justifie l'action (en italiques) est susceptible d'être assertée par un énonciateur rapporté : la particule énonciative ved' ${ }^{16}$ et les qualificatifs (takie grubosti) relèvent du point de vue subjectif. Cependant, tout comme c'est le cas dans [5] et [6], il n'y a pas ici de décrochage énonciatif. Le lien logique avec le plan du récit est très fort : il s'agit de l'interprétation d'une action, d'un événement, ce qui correspond à la définition du point de vue selon Rabatel citée plus haut; de plus, le prétérit du verbe slyšat' maintient l'ancrage de la phrase dans le récit. Aussi, la séquence en question est trop courte car, en général, il faut plus d'une phrase pour quitter la zone du récit ; les courts énoncés, malgré les traces expressives, s'inscrivent plutôt comme commentaires (empathiques ou ironiques) du narrateur sur la manière de percevoir du personnage. Ainsi, même si cette phrase est dans l'ensemble potentiellement verbalisable, l'insuffisance des marqueurs discursifs l'empêche de quitter la zone de la narration.

\subsection{Du point de vue au DIL. Effet de DIL}

L'exemple suivant montre une continuité dans les différentes étapes de l'articulation de la pensée et constitue à ce sujet un développement de la technique argumentative que nous avons vue dans l'exemple précédent de Čexov :

[8] [En ouvrant son journal du matin, Ivan Alexandrovitch Petrov découvre qu'il n'arrive plus à voir distinctement les lignes du texte. Il fait le constat terrible que sa vue baisse et que la quarantaine est le présage de bien des malheurs dans la vie d'un homme.] Отец остался один со своей газетой.

[i] Иван Александрович Петров. Дворянин. Домовладелец. Счастливый отец семейства. Радостно празднует в кругу любящих домашних - разжигал он себя.[ii] Он и сам понимал, что это глупо и чисто случайно вышло так, что все оказались в одном углу комнаты, а он в другом, и никто ведь не знает, что с ним стряслось только что. [iii] Но всё-таки, при известной чуткости, мог бы кто-нибудь остановиться и взглянуть на отца?

(И. Ратушинская, Одессить)

Une fois le sujet de conscience, l'énonciateur potentiel posé, nous entrons dans l'espace perceptif d'autrui ; le passage s'engage comme récit - on présente au lecteur un nouveau personnage, mais le commentaire du narrateur razžigal on sebja « enrageait-il » à la fin de la séquence [i] indique explicite-

15. Cf. pour l'analyse de cet exemple S. Rigato 1998: 168 : l'extrait en italique est défini comme DIL. 16. Sur la particule ved', on peut se référer à PAILlARD et MARKOWICZ 1986. 
ment qu'il s'agit du discours intérieur d'un père de famille qui fait la description de la scène comme vue de l'extérieur. Tout cet extrait joue sur la transgression des limites entre le récit, la représentation de la conscience (au psycho-récit) et le discours intérieur : en [ii], on peut parler du récit en focalisation extérieure - il s'agit de la description empathique au psycho-récit d'un état mental du personnage, mais sans décrochage énonciatif qui ferait basculer la narration dans l'énonciation. De plus, le prétérit ponimal « comprenait » qui introduit une subordonnée mentionnant une prise de conscience d'un fait (vision impartiale de la scène : personne n'est responsable de son sentiment de solitude) rattache davantage la phrase au plan du récit. Dans [iii], la connaissance de ce fait est ramenée au niveau réflexif par le biais d'une question rhétorique qui comporte des contre-arguments à cette vision objective de la scène.

Ainsi, nous avons dans cet extrait un exemple des entrelacs entre la représentation du point de vue, du psycho-récit et du discours intérieur qui, grâce aux liens divers qui unissent ces divers plans (connecteurs, syntaxe, éléments subjectifs) créent dans l'ensemble un effet de DIL, même s'il ne peut s'agir ici du discours verbalisable dans sa totalité sous cette forme : lorsqu'il est question des divers états mentaux complexes, on ne peut en donner qu'une représentation, possible seulement dans le récit de fiction.

Encore un exemple qui montre la manière dont sont intriqués les commentaires du narrateur, posés en amont dans le texte et très mimétiques du discours de l'énonciateur rapporté, et ce que l'on interprète sans difficulté comme DIL (en italiques) :

[9] Алина Федоровна, мать Левки Шулепы, могла потыкать вилкою в кусок торта и отодвинуть его, сказав: «По-моему, торт несвеж», - и торт уносили. [i] Когда это случилось впервые, Глебов про себя поразился. [iі] Как может быть торт несвеж? Ему показалось это совершенной нелепостью. [iii] У него дома торт появлялся редко, ко дню рождения, съедали его быстро, и никому не приходило в голову выяснить, свеж он или несвеж. [iv] Он всегда был свеж, великолепно свеж, особенно такой пышный, с розовыми цветами из крема.

(Ю. Трифонов, Дом на набережной)

Ici, nous avons également la représentation de la pensée qui mêle le verbal et le non-verbal : d'abord, en [i], l'énonciateur potentiel est posé par un énoncé qui mentionne sa réaction (l'étonnement) et crée une demande d'explicitation (déclencheur classique du DIL) ; ensuite, en [ii], nous avons un développement du comportement de l'énonciateur sous forme de question que l'on attribue à l'énonciateur rapporté (Glebov). Le [iii] constitue, lui, une description de ce que le personnage peut vraisemblablement passer en revue comme souvenirs, sans pour autant les verbaliser, mais la représentation qui est faite de ce processus mental est très vraisemblable du point de vue langagier. En revanche, l'alignement des repères temporels sur le plan du récit est un argument qui amène à attribuer le (iii) à la zone de narration, même si celle-ci est « contaminée » par les intonations d'autrui : «svež on ili nesvež» sont des paroles citées, filtrées par le prisme de la conscience d'autrui ; somme toute, 
nous avons affaire à l'expression du point de vue d'autrui ${ }^{17}$. Le (iv) est énoncé en termes qui permettent de rebasculer dans la zone d'énonciation, le nombre d'éléments subjectifs étant suffisant pour créer un décrochage énonciatif. Tout comme dans le texte de Ratušinskaja, ici l'agencement des séquences que l'on a définies comme point de vue et celles qui représentent le discours créent un effet de DIL dans l'ensemble.

\section{Conclusions}

Le discours indirect libre paraît comme un terme très imprécis, influencé par le contexte littéraire qui a favorisé son emploi : popularisé par le roman réaliste, le DIL sert la plupart du temps à désigner l'ensemble des manifestations de la subjectivité (conscience, perceptions). Il se présente ainsi comme une seule et grande catégorie entre deux pôles : d'un côté, le récit, la zone du narrateur, de l'autre, le discours, la zone du personnage. Or, il existe plus d'un procédé qui fait passer d'un pôle à l'autre ; en faire une impasse, en désignant par DIL, grosso modo, toute séquence qui ne peut plus être entièrement validée par le narrateur, dont le discours est censé être dépourvu de subjectivité, est une erreur, ne serait-ce que parce qu' on peut avoir un narrateur « empathique » qui va imiter parfois le discours oral, sans que celui-ci puisse être attribué à un énonciateur concret. Il est souvent difficile de juger ce qui relève du véritable discours représenté en l'absence d'extrait suffisamment long; parfois, nous avons besoin de plusieurs paragraphes du récit pour pouvoir juger de l'instance du narrateur, des caractéristiques de son discours et de son rapport à l'univers représenté.

La difficulté majeure de l'analyse du DIL est liée aux cas où ce terme désigne les pensées. Il est souvent permis de douter de la possibilité de l'existence du discours lui-même : dans ces cas-là, le terme «DIL » paraît abusif, opacifiant la réalité langagière : il est nécessaire alors d'introduire d'autres termes, comme point de vue et « effet de DIL », pour décrire les pensées. Des cas de représentation de l'univers des perceptions, des réactions spontanées, des émotions amènent le linguiste à réfléchir à la fois sur les étapes cognitives qui accompagnent la naissance du discours intérieur et sur le processus d'écriture qui permettra au lecteur d'accéder à l'univers normalement inaccessible : on doit alors avancer avec précaution dans le labyrinthe des faux-semblants, se plaçant en plein cœur $\mathrm{du}$ - on prend le risque de choisir ce terme - « flux de pensée $»$.

En conclusion, nous dirons que lorsqu'il s'agit de rendre compte de la représentation de la pensée, processus partiellement verbal, le terme «DIL » est plutôt opacifiant, insuffisant par lui seul pour décrire les différents stades de construction de l'espace énonciatif d'autrui.

17. USPENSKIJ 1973/2000, p. 78 note que dans ces cas-là, on se réfère à un monologue conventionnel qui n'existe pas en réalité, mais qui peut être représenté en principe. 


\section{BIBLIOGRAPHIE}

ARTYUSHKINA O. 2010. Le discours indirect libre en russe, thèse de doctorat <http://www.e-sorbonne.fr/sites/www.e-sorbonne.fr/files/theses/Art11.these_.integrale.pdf> Axmanova O. 1966. Slovar' lingvističeskix terminov, M., Sovetskaja enciklopedija. Banfield A. 1982/1995. Phrases sans parole, Paris, Éd. du Seuil.

Benveniste É. 1970/1974. «L'appareil formel de l'énonciation ». Problèmes de linguistique générale 2, Paris, Gallimard.

CERQuiligni B. 1984. « Le style indirect libre et la modernité », in Langages, vol. 19, $\mathrm{n}^{\circ}$ 73, pp. 7-16.

Cohn D. 1981. La transparence intérieure, Paris, Éditions du Seuil.

DE MATTIA-VIVIÈs M. 2006. Le discours indirect libre au risque de la grammaire : le cas de l'anglais, Presses universitaires de Provence (PUP).

FONTAINE J. 1977. « Discours direct et discours indirect», in $I I^{e}$ Colloque de linguistique russe, Paris, Institut d'études slaves, pp. 349-361.

Genette G. 1969. Figures II, Paris, Éditions du Seuil.

ID. 1972. Figures III, Paris, Éditions du Seuil.

Gvozdev A. N. 1965. Očerki po stilistike russkogo jazyka, M., Prosveščenie.

Grammatika russkogo jazyka. 1954, t. 2, M., Izd. AN SSSR.

Hanote S., ChuQuet H. 2004. Who's speaking, please? Le discours rapporté, Paris, Ophrys.

LEONT'EV K. 1911. O romanax grafa L. N. Tolstogo: analiz, stil' i vejanie, M.

MANN Ju. 1992. «Ob evolucii povestvovatel'nyx form (vtoraja polovina XIX v.) », in Serija literatury i jazyka, t. 51, n ${ }^{0}$ 1, pp.40-59.

MARNETTE S. 2002. «Étudier les pensées rapportées en français parlé : mission impossible ? », in Faits de langues, n 19, pp. 211-221.

PAILlARD D. et MARKOVICZ D. 1986. Le partage du savoir ou l'ignorance n'est pas un argument : à propos de la particule ved', in les Particules énonciatives en russe contemporain, Paris, Institut d'études slaves, vol. 1, pp. 89-125.

PEŠKOVSKIJ A. 1920/1956. Russkij sintaksis v naučnom osveščenii, M, Gos. Izd.

PERnot C. 2008. « Le discours indirect libre médiéval : épistémologie et enjeux méthodologiques ", Université de Nancy <http://revendie.free.fr>

RABATEl A. 2001. " Les représentations de la parole intérieure. Monologue intérieur, discours direct et indirect libres : point de vue », Langue française, vol. 132, $\mathrm{n}^{\circ}$ 1, pp. 72-95 <http://www.persee.fr>

RozentaL' D. 1976. Slovar'-spravočnik lingvističeskix terminov, M., Prosveščenie.

Russel B. 1940. An Inquiry into Meaning and Truth, New York, Norton; trad. fr. Ph. Devaux, Signification et vérité, Paris, Flammarion, 1990.

Rigato S. 1998. «Nesobstvenno-prjamaja reč' i eë formy vo vtoroj časti romana Ju. K. Oleši Zavist'», in Slavica tergestina, 6, pp. 163-196.

SoKolova L. 1968. Nesobstvenno-avtorskaja reč' (nesobstvenno-prjamaja) reč' kak stilističeskaja kategorija, dissert. kand. filolog. nauk, Tomsk.

ŠMID, V. 2003. Narratologija, M., Jazyki slavjanskoj kul'tury.

UsPenskij B. 1973/2000. Poètika kompozicii, SPb, Azbuka.

VEYRENC J. 1982. « Note sur le style indirect libre (SIL) dans les fables de Krylov », in Revue des études slaves, t. 54, fasc. 3, pp. 437-453.

VološINOV V. 1929/1998. Marksizm i filosofija jazyka, M., Labirint. 


\section{РЕЗЮМЕ}

Появление понятия несобственно-прямой речи (НПР) связано с зарождением в литературе тенденции к психологизации и связанному с ней интересу к изображению внутренного мира персонажей, где особое место занимает внутренняя речь и мыслительный процесс.

Разнообразие примеров НПР удивляет своей неоднородностью и тем самым ставит под вопрос само использование данного лингвистического термина, который применим к любым выражениям речи, но также эмоций, субъективности в нарративе, несовместимыми с позицией повествователя. В статье представляется критика такого определения НПР, поскольку само понятие становится формально неопределенным.

В статье развивается идея разграничения между изображением собственно внутренней речи и процессом становления мысли, который включает в себя не только вербальную стадию, но и невербальную, состояющую из зрительных впечатлений, ремеморизации и др. Таким образом, в таком контексте применение существующего термина проблематично, так как он отсылает к вербальной стадии выражения мысли. В таких случаях более применительным становится термин точки зрения, а также предлагаемое в статье понятие «эффекта несобственно-прямой речи».

\section{SUMMARY}

The appearance of the concept of free indirect discourse (FID) is connected with the tendancy of psychologisation in literature which involves the interest in the representation of interior discourse and mental process.

The variety of examples of FID respresents a great heterogeneity which renders hypothetical the very use of this linguistic term. Indeed, FID is used to refer not only to the indirect (or interior) discourse, but also to represent any expession of emotion and subjectivity uncompatible with the narrator's position. The paper gives a critical review of such approach of FID, which shows the weekness of the formal definition of the concept.

The author of this paper develops the idea of differentiation between the representation of the interior discourse and the mental process of thought formulation which involves not only a verbal stage, but a non verbal one as well, composed of visual perception, rememoration, etc. In this context the use of this term is problematic because it refers only to a verbal aspect of thought. In such cases the term "point of view" and the notion of "FID effect" proposed by the author are more appropriate. 